\title{
CONDITIONS FOR ESCAPE AND RETENTION
}

\author{
J. S. GRIFFITH
}

Lakehead University, Thunder Bay, Ontario, Canada P7B SEI

\begin{abstract}
The methods used in deriving conditions for escape, retention and containment for the threebody problem are applied to the $n$-body case, and similar conditions are obtained. In the $n$-body problem less stringent conditions are derived, and in the case of retention a further condition is imposed.
\end{abstract}

\section{Introduction}

The classification of the types of motion of the general three-body problem as the time becomes infinite is well known (Chazy, 1922), but the determination of the type of motion for any given initial conditions is difficult. Standish $(1971,1972)$ has given sufficient conditions for the retention or escape of a member of a three-body system. These conditions were strengthened by Griffith and North (1973), using a similar technique to that of Standish. Yoshida (1972), with an alternative approach, obtained slightly better conditions for escape, but with a much lengthier derivation. In this paper the results and methods of Griffith and North for the three-body problem are recapitulated, expanded and applied to the general $N$-body problem. Section 2 deals with escape or retention in the three-body problem, Section 3 with a new containment theorem for the three body problem, while Section 4 extends the results to the general $N$-body problem. All results apply to motion of the escaping body with respect to the barycentre of the remaining bodies.

\section{Three-Body Escape or Retention}

The three masses are denoted $m_{a}, m_{b}, m_{c}$ with $r$ the distance of mass $m_{a}$ from mass $m_{b}, \varrho$ the distance of mass $m_{c}$ from the centre of mass of $m_{a}$ and $m_{b}$. The case where $m_{c}$ passes directly between $m_{a}$ and $m_{b}$ is avoided by the condition $r \leqslant \varrho_{a c}, \varrho_{b c}$ where $\varrho_{a c}, \varrho_{b c}$ are respectively the distances between $m_{a}$ and $m_{c}$ and between $m_{b}$ and $m_{c}$.

The equation of motion for $\varrho$ is

$$
\ddot{\varrho}=g_{2}^{2}\left(p_{\theta}^{2} / \varrho^{3} \cos ^{2} \phi+p_{\phi}^{2} / \varrho^{3}\right)+g_{2}(\partial F / \partial \varrho),
$$

where

$$
g_{2}=M / m_{c}\left(m_{a}+m_{b}\right), \quad M=m_{a}+m_{b}+m_{c}
$$

and

$$
F=G\left(\frac{m_{a} m_{b}}{r}+\frac{m_{a} m_{c}}{\varrho_{a c}}+\frac{m_{c} m_{b}}{\varrho_{b c}}\right)
$$

The previous proofs of Standish depend upon obtaining upper and lower bounds 
for $\ddot{\varrho}$, multiplying by $\dot{\varrho}$ and integrating with respect to time. This procedure will be followed here, with

$$
M_{a}=m_{a} /\left(m_{a}+m_{b}\right) \text { and } M_{b}=1-M_{a} .
$$

\section{ESCAPE THEOREM}

If at some time, $t_{0}$

(i) $\varrho_{0}>r_{*}$ (the maximum separation of $m_{a}, m_{b}=G\left(m_{a} m_{b}+m_{b} m_{c}+m_{c} m_{a}\right) /|E|$, where $E$ is the total energy),

(ii) $\dot{\varrho}_{0}>0$, and

$$
\dot{\varrho}_{0}^{2} / 2>G M\left[\frac{1}{\varrho_{0}}+\frac{M_{a} M_{b} r_{*}}{\varrho_{0}^{2}}\left\{\frac{M_{b}}{\varrho_{0}-M_{b} r_{*}}+\frac{M_{a}}{\varrho_{0}-M_{a} r_{*}}\right\}\right],
$$

then $\varrho \rightarrow \infty$ as $t \rightarrow \infty$.

Now

$$
\begin{aligned}
\ddot{\varrho} \geqslant g_{2} \frac{\partial F}{\partial \varrho} & =G M \frac{\partial}{\partial \varrho}\left(\frac{M_{a}}{\varrho_{a c}}+\frac{M_{b}}{\varrho_{b c}}\right)= \\
& =G M \frac{\partial}{\partial \varrho}\left[\frac{1}{\varrho}+\frac{M_{a} M_{b}}{\varrho} \sum_{i=2}^{\infty}\left(M_{b}^{i-1}+\left(-M_{a}\right)^{i-1}\right)\left(\frac{r}{\varrho}\right)^{i} P_{i}(q)\right],
\end{aligned}
$$

where the $P_{i}(q)$ are Legendre polynomials,

$$
\begin{aligned}
& =G M \frac{\partial}{\partial \varrho}\left[\frac{1}{\varrho}+\frac{M_{a} M_{b}}{\varrho} \sum_{i=2}^{\infty}\left(\left(\frac{M_{b} r}{\varrho}\right)^{i} \frac{P_{i}(q)}{M_{b}}+\left(\frac{-M_{a} r}{\varrho}\right)^{i} \frac{P_{i}(q)}{M_{a}}\right)\right]= \\
& =G M\left[-\frac{1}{\varrho^{2}}-\frac{M_{a} M_{b}}{\varrho} \sum_{i=2}^{\infty}(i+1)\left(\left(\frac{M_{b} r}{\varrho}\right)^{i}\left(\frac{P_{i}(q)}{M_{b}}\right)+\left(\frac{-M_{a} r}{\varrho}\right)^{i} \frac{P_{i}(q)}{M_{a}}\right)\right] .
\end{aligned}
$$

Our aim is to establish upper and lower bounds to this expression. The sharpest conditions may be found by determining for what value of $q$ this attains a maximum or minimum, but this appears difficult as differentiation with respect to $q$ yields $\sin q=0$ or $\cos q$ as the root of a 7 th-order equation. Only for equal masses $\left(m_{a}=m_{b}\right)$ does the equation have simpler roots. Cruder estimates of the bounds are available, for as $\left|P_{i}(q)\right| \leqslant 1$,

$$
\ddot{\varrho} \geqslant G M\left[-\frac{1}{\varrho^{2}}-\frac{M_{a}}{\varrho^{2}} \sum_{i=2}^{\infty}(i+1)\left(\frac{M_{b} r}{\varrho}\right)^{i}-\frac{M_{b}}{\varrho^{2}} \sum_{i=2}^{\infty}(i+1)\left(\frac{M_{a} r}{\varrho}\right)^{i}\right] .
$$

It is at this point that my approach diverges from that of Standish, for he used the additional approximation that $\left|M_{b}^{i-1}+\left(-M_{a}\right)^{i-1}\right| \leqslant 1$ and hence obtained a simpler, less precise, expression.

Using $r_{*}$ as the maximum value of $r$, we have

$$
\ddot{\varrho} \geqslant G M\left[-\frac{1}{\varrho^{2}}-M_{a} \sum_{i=2}^{\infty} \frac{(i+1)\left(M_{b} r_{*}\right)^{i}}{\varrho^{i+2}}-M_{b} \sum_{i=2}^{\infty} \frac{(i+1)\left(M_{a} r_{*}\right)^{i}}{\varrho^{i+2}}\right] .
$$


For escape, assume $\dot{\varrho}>0$ in some interval of time $\left(t_{0}, t_{1}\right)$, where $t_{1}>t_{0}$, then

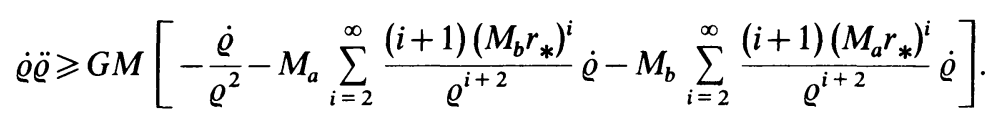

Integrating from $t_{0}$ to $t_{1}$ gives

$$
\frac{1}{2} \dot{\varrho}_{1}^{2} \geqslant G M\left[\frac{1}{\varrho_{1}}+\frac{M_{a}\left(M_{b} r_{*}\right)^{2}}{\varrho_{1}^{2}\left(\varrho_{1}-M_{b} r_{*}\right)}+\frac{M_{b}\left(M_{a} r_{*}\right)^{2}}{\varrho_{1}^{2}\left(\varrho_{1}-M_{a} r_{*}\right)}\right]+K,
$$

where

$$
K=\frac{1}{2} \dot{\varrho}_{0}^{2}-G M\left[\frac{1}{\varrho_{0}}+\frac{M_{a}\left(M_{b} r_{*}\right)^{2}}{\varrho_{0}^{2}\left(\varrho_{0}-M_{b} r_{*}\right)}+\frac{M_{b}\left(M_{a} r_{*}\right)^{2}}{\varrho_{0}^{2}\left(\varrho_{0}-M_{a} r_{*}\right)}\right],
$$

and $\dot{\varrho}_{1}, \varrho_{1}$ are respectively the values of $\underline{\varrho}, \varrho$ at time $t_{1}$.

For $K \geqslant 0$, then $\dot{\varrho}_{1}^{2}>0$ for all finite values of $\varrho_{1}$. Then $\dot{\varrho}$ remains positive for all time and $\frac{1}{2} \dot{\varrho}^{2} \geqslant K$ for all $t>t_{0}$ or $\varrho \geqslant \sqrt{ }(2 K)\left(t-t_{0}\right)+\varrho_{0}$. So $\varrho \rightarrow \infty$ as $t \rightarrow \infty$ if $K>0$ and escape occurs. Hence the escape theorem is true.

The value for condition (iii) given by Standish differs from this with the difference decreasing asymptotically as $\varrho_{0}^{-4}$. For small values of $\varrho$, this revised value of $K$ is markedly better than that of Standish.

To compare these conditions in detail, we follow Standish with $\alpha=\varrho_{0} / r_{*}, G=M=$ $=r_{*}=1$ to obtain

$$
\begin{aligned}
G M\left[\frac{1}{\varrho_{0}}+\frac{M_{a} M_{b} r_{*}^{2}}{\varrho_{0}^{2}}\left\{\frac{M_{b}}{\varrho_{0}-M_{b} r_{*}}+\frac{M_{a}}{\varrho_{0}-M_{a} r_{*}}\right\}\right]= \\
=\frac{1}{\alpha}+\frac{M_{a} M_{b}}{\alpha^{2}}\left(\frac{M_{b}}{\alpha-M_{b}}+\frac{M_{a}}{\alpha-M_{a}}\right),
\end{aligned}
$$

compared to Standish's expression $1 / \alpha+M_{a} M_{b} / \alpha^{2}(\alpha-1)$.

The numerical comparison is given in Table $I$, with the upper entry being the preceding expression, the middle entry that of Standish and the lower that of Tevzadze (1962). It is seen that our condition is superior to the others in all cases. Tevzadze used

$$
G M\left[\frac{M_{b}}{\varrho_{0}-M_{a} r_{*}}-\frac{M_{a}}{\varrho_{0}-M_{b} r_{*}}\right]=\frac{M_{b}}{\alpha-M_{a}}+\frac{M_{a}}{\alpha-M_{b}} .
$$

If $\varrho<r_{*}$ the preceding analysis will not work. Expansion in powers of $\varrho / r$ is not effective, as the result hinges on the right hand side of the inequality reversing sign on integration and becoming positive, thus enabling us to assert that $K>0$. Without inverse powers of $\varrho$ to integrate, this reversal is not possible. There are also difficulties in expansions in the intermediate region $r<\varrho<r_{*}$.

For equal masses,

$$
g_{2} \frac{\partial F}{\partial \varrho}=\frac{G M}{2} \frac{\partial}{\partial \varrho}\left(\frac{1}{\varrho_{a c}}+\frac{1}{\varrho_{b c}}\right)
$$


TABLE I

Comparison of conditions (iii)

\begin{tabular}{llll}
\hline$x$ & $M_{a}=0.1$ & 0.3 & 0.5 \\
\hline 1.1 & 1.2512 & 1.2779 & 1.2534 \\
& 1.6529 & 2.6446 & 2.9752 \\
1.3 & 1.4000 & 1.6250 & 1.6667 \\
& 0.8935 & 0.9515 & 0.9541 \\
& 0.9467 & 1.1834 & 1.2623 \\
1.5 & 1.0000 & 1.2000 & 1.2500 \\
& 0.7295 & 0.7717 & 0.7778 \\
& 0.7467 & 0.8533 & 0.8889 \\
2.0 & 0.8095 & 0.9583 & 1.0000 \\
& 0.5196 & 0.5375 & 0.5417 \\
& 0.5225 & 0.5525 & 0.5625 \\
3.0 & 0.5646 & 0.6425 & 0.6667 \\
& 0.3380 & 0.3430 & 0.3444 \\
& 0.3383 & 0.3450 & 0.3472 \\
5.0 & 0.3580 & 0.3897 & 0.4000 \\
& 0.20086 & 0.2019 & 0.2022 \\
& 0.20090 & 0.2021 & 0.2025 \\
10.0 & 0.20806 & 0.2187 & 0.2222 \\
& 0.100098 & 0.10022 & 0.10026 \\
& 0.100100 & 0.10023 & 0.10028 \\
& 0.101898 & 0.10442 & 0.10526
\end{tabular}

where

$$
\begin{aligned}
& \varrho_{a c}^{2}=\varrho^{2}+\frac{1}{2} r+\varrho r \cos q, \\
& \varrho_{b c}^{2}=\varrho^{2}+\frac{1}{2} r-\varrho r \cos q .
\end{aligned}
$$

Using $(\partial / \partial q)\left(g_{2}(\partial F / \partial \varrho)\right)=0$ we find $\sin q=0$ or

$$
\frac{2 \varrho^{2}-\left(\frac{1}{2} r\right)^{2}+\frac{1}{2} \varrho r \cos q}{2 \varrho^{2}-\left(\frac{1}{2} r\right)^{2}-\frac{1}{2} \varrho r \cos q}=\frac{\left(\varrho^{2}+\left(\frac{1}{2} r\right)^{2}+\varrho r \cos q\right)^{5 / 2}}{\left(\varrho^{2}+\left(\frac{1}{2} r\right)^{2}-\varrho r \cos q\right)^{5 / 2}} .
$$

This last equation gives $\cos q=0$ or

$$
\begin{aligned}
\frac{\gamma^{6} \cos ^{6} q}{4} & +\gamma^{4} \cos ^{4} q\left(\left(2 \varrho^{2}-\left(\frac{1}{2} r\right)^{2}\right)^{2}+5\left(2 \varrho^{2}-\left(\frac{1}{2}\right)^{2}\right)\left(\varrho^{2}+\left(\frac{1}{2} r\right)^{2}\right)+\right. \\
& \left.+5\left(\varrho^{2}+\left(\frac{1}{2} r\right)^{2}\right)^{2}\right)+\gamma^{2} \cos ^{2} q\left(10\left(\varrho^{2}+\left(\frac{1}{2} r\right)^{2}\right)^{2}\left(2 \varrho^{2}-\left(\frac{1}{2} r\right)\right)^{2}+\right. \\
& \left.+10\left(\varrho^{2}+\left(\frac{1}{2} r\right)^{2}\right)^{3}\left(2 \varrho^{2}-\left(\frac{1}{2} r\right)^{2}\right)+\left(\frac{5}{2}\right)\left(\varrho^{2}+\left(\frac{1}{2} r\right)^{2}\right)^{4}\right)+ \\
& +\left(2 \varrho^{2}-\left(\frac{1}{2} r\right)^{2}\right)\left(\varrho^{2}+\left(\frac{1}{2} r\right)^{2}\right)^{5}+\left(2 \varrho^{2}-\left(\frac{1}{2} r\right)^{2}\right)^{2}\left(\varrho^{2}+\left(\frac{1}{2} r\right)^{2}\right)^{4}=0,
\end{aligned}
$$

where $\gamma=\varrho r$.

As $\varrho>r / 2 \sqrt{ } 2$ the only solutions are $\cos q=\neq 1$, giving motion along the perpendicular to the lines of centres. Using the extreme values of $\cos q= \pm 1$, we have

$$
\ddot{\varrho} \geqslant-\frac{G M\left(\varrho^{2}+\left(\frac{1}{2} r\right)^{2}\right)}{\left(\varrho^{2}-\left(\frac{1}{2} r\right)^{2}\right)^{2}} \geqslant \frac{G M\left(\varrho^{2}+r_{*}^{2}\right)}{\left(\varrho^{2}-r_{*}^{2}\right)^{2}} .
$$


On multiplication by $\dot{\varrho}$ and integrating we may compare this new value of $K$ (equal masses) with the value of $K$ from the escape theorem with $M_{a}=M_{b}=\frac{1}{2}$, which is

$$
\frac{1}{2} \dot{\varrho}_{0}^{2}-G M\left(\frac{1}{\varrho_{0}}+\frac{1}{4} \frac{r_{*}^{2}}{\varrho_{0}^{2}\left(\varrho_{0}-r_{*} / 2\right)}\right) \text {. }
$$

\section{RETENTION THEOREM}

If the mutual distance between the bodies of mass $m_{a}$ and $m_{b}$ is bounded by $r_{*} \geqslant r \geqslant$ $\geqslant r_{*} m_{a} m_{b} /\left(m_{a} m_{b}+m_{b} m_{c}+m_{c} m_{a}\right)$ and if at some time $t_{0}$

(i) $\varrho_{0}>r_{*}$,

(ii) $\dot{\varrho}_{0}>0$,

(iii)

$$
\begin{aligned}
\frac{1}{2} \dot{\varrho}_{0}^{2}<\frac{G M}{\varrho_{0}} & -\frac{G M M_{a} M_{b}}{\varrho_{0}^{2}} \frac{r_{*}^{2}}{\varrho_{0}-r_{*}}-\frac{2 G g_{2} m_{c}}{r_{*}}\left\{\frac{m_{a}}{M_{b}} \ln \left(1+M_{b} r_{*} / \varrho_{0}\right)+\right. \\
& \left.+\frac{m_{b}}{M_{a}} \ln \left(1+M_{a} r_{*} / \varrho_{0}\right)\right\},
\end{aligned}
$$

then $m_{c}$ is retained by the system, at least until $\varrho$ becomes negative and $\varrho$ becomes less than $r_{*}$.

The differential equation for $\varrho$ (Equation (1)) may be written

$$
\ddot{\varrho}=|\boldsymbol{\varrho} \wedge \dot{\boldsymbol{\varrho}}|^{2} / \varrho^{3}+g_{2}(\partial F / \partial \varrho) \text {. }
$$

Standish deduced a time independent upper bound for $|\boldsymbol{\varrho} \wedge \dot{\boldsymbol{\varrho}}|^{2}$ before multiplication by $\dot{\varrho}$ and integration. One way of refining his conditions is to use

$$
\dot{\boldsymbol{Q}}^{2}=\left\{(\boldsymbol{Q} \dot{\boldsymbol{Q}})^{2}+(\boldsymbol{Q} \wedge \dot{\mathbf{e}})^{2}\right\} / \varrho^{2}=\dot{\varrho}^{2}+(\boldsymbol{\varrho} \wedge \dot{\mathbf{Q}})^{2} / \varrho^{2},
$$

so that

$$
\frac{1}{\varrho^{3}}|\varrho \wedge \dot{\varrho}|^{2}=\frac{\dot{\varrho}^{2}-\dot{\varrho}^{2}}{\varrho} \leqslant \frac{(E+F) 2 g_{2}-\dot{\varrho}^{2}}{\varrho}
$$

where $E$ is the total energy of the system and $F$ the potential.

The difficulty of bounding this expression is the presence of the $m_{a} m_{b} / r$ term in $F$, which may become large if the minimum interparticle distance is small. A set of conditions not yet fully utilized are those contained in the energy integral. From

$$
E=\frac{1}{2 g_{1}} \dot{\mathbf{r}}^{2}+\frac{1}{2 g_{2}} \dot{\mathbf{Q}}^{2}-F,
$$

we have $E+F \geqslant 0$ (here $\left.g_{1}=\left(m_{a}+m_{b}\right) / m_{a} m_{b}\right)$, i.e.

$$
E \geqslant-F=-G\left(\frac{m_{a} m_{b}}{r}+\frac{m_{a} m_{c}}{\varrho_{a c}}+\frac{m_{b} m_{c}}{\varrho_{b c}}\right) \geqslant-G\left(m_{a} m_{b}+m_{a} m_{c}+m_{b} m_{c}\right) / r .
$$

If $E$ is negative,

$$
r \leqslant r_{*}=G\left(m_{a} m_{b}+m_{a} m_{c}+m_{b} m_{c}\right) /|E|
$$


which was noted by Standish. We have from

$$
E \geqslant-G\left(\frac{m_{a} m_{b}}{r}+\frac{m_{a} m_{c}}{\varrho_{a c}}+\frac{m_{b} m_{c}}{\varrho_{b c}}\right)
$$

the condition that, if $\varrho$ does become arbitrarily large, $E \geqslant G m_{a} m_{b} / r$ or $r \leqslant r_{*} m_{a} m_{b} /$ $\left(m_{a} m_{b}+m_{a} m_{c}+m_{b} m_{c}\right)$.

If $\varrho$ is allowed to become arbitrarily large, we require

$$
\frac{m_{a} m_{b}+m_{a} m_{c}+m_{b} m_{c}}{r_{*}} \leqslant \frac{m_{a} m_{b}}{r}
$$

i.e.

$$
r \leqslant \frac{r_{*} m_{a} m_{b}}{m_{a} m_{b}+m_{a} m_{c}+m_{b} m_{c}}
$$

Let us assume that $r_{*} \geqslant r \geqslant r_{*} m_{a} m_{b} /\left(m_{a} m_{b}+m_{a} m_{c}+m_{b} m_{c}\right)$. Thus, to keep the third body within the system, we do not allow the other two bodies to become so close as to allow their lost potential energy to manifest itself in the escape of the third body. With this lower bound on $r$ we return to

$$
\ddot{\varrho}=|\boldsymbol{\varrho} \wedge \dot{\boldsymbol{\varrho}}|^{2} / \varrho^{3}+g_{2}(\partial F / \partial \varrho), \quad \text { and } \quad|\boldsymbol{\varrho} \wedge \dot{\boldsymbol{\varrho}}|^{2} / \varrho^{3} \leqslant 2 g_{2}(E+F) / \varrho,
$$

to obtain

$$
\begin{aligned}
\ddot{\varrho} \leqslant 2 g_{2}\{E & \left.+G\left(\frac{\left(m_{a} m_{b}+m_{a} m_{c}+m_{b} m_{c}\right)}{r_{*}}+\frac{m_{a} m_{c}}{\varrho-M_{b} r_{*}}+\frac{m_{b} m_{c}}{\varrho-M_{a} r_{*}}\right)\right\} / \varrho+ \\
+G M & {\left[-\frac{1}{\varrho^{2}}+M_{a} M_{b} \sum_{i=2}^{\infty}(i+1) \frac{r_{*}^{i}}{\varrho^{i+2}}\right] . }
\end{aligned}
$$

Assume that $\dot{\varrho}>0$ for all time $t>t_{0}$. Then, multiplying the expression for $\ddot{\varrho}$ by $\dot{\varrho}$ and integrating from $t_{0}$ to $t$, gives

$$
\begin{aligned}
\frac{1}{2}\left(\dot{\varrho}_{1}^{2}-\dot{\varrho}_{0}^{2}\right) \leqslant & 2 g_{2}\left\{E+G \frac{\left(m_{a} m_{b}+m_{a} m_{c}+m_{b} m_{c}\right)}{r_{*}}\right\} \ln \frac{\varrho_{1}}{\varrho_{0}}- \\
& -\frac{2 g_{2} m_{a} m_{c} G}{M_{b} r_{*}}\left[\ln \left(\frac{\varrho-M_{b} r_{*}}{\varrho}\right)\right]_{0}^{1}- \\
& -\frac{2 g_{2} m_{b} m_{c} G}{M_{a} r_{*}}\left[\ln \left(\frac{\varrho-M_{a} r_{*}}{\varrho}\right)\right]_{0}^{1}+\left[\frac{G M}{\varrho}-\sum_{i=2}^{\infty} G M M_{a} \frac{M_{b} r_{*}^{i}}{\varrho^{i+1}}\right]_{0}^{1} .
\end{aligned}
$$

Now $E+G\left(m_{a} m_{b}+m_{c}+m_{b} m_{c}\right) / r_{*}=0$ giving

$$
\begin{gathered}
\frac{1}{2} \dot{\varrho}_{1}^{2} \leqslant\left[\frac{1}{2} \dot{\varrho}_{0}^{2}+\frac{2 g_{2} m_{a} m_{c} G}{M_{b} r_{*}} \ln \left(\frac{\varrho_{0}-M_{b} r_{*}}{\varrho_{0}}\right)+\frac{2 g_{2} m_{b} m_{c} G}{M_{a} r_{*}} \ln \left(\frac{\varrho_{0}-M_{a} r_{*}}{\varrho_{0}}\right)-\right. \\
\left.-\frac{G M}{\varrho_{0}}+\frac{G M M_{a} M_{b} r_{*}^{2}}{\varrho_{0}^{2}\left(\varrho_{0}-r_{*}\right)}\right]-\frac{2 g_{2} m_{a} m_{c} G}{M_{b} r_{*}} \ln \left(\frac{\varrho_{1}-M_{b} r_{*}}{\varrho_{1}}\right)-
\end{gathered}
$$




$$
-\frac{2 g_{2} m_{b} m_{c} G}{M_{a} r_{*}} \ln \left(\frac{\varrho_{1}-M_{a} r_{*}}{\varrho_{1}}\right)+\frac{G M}{\varrho_{1}}-\frac{G M M_{a} M_{b}}{\varrho_{1}^{2}} \frac{r_{*}^{2}}{\left(\varrho_{1}-r_{*}\right)}
$$

so that

$$
\begin{aligned}
\frac{1}{2} \dot{\varrho}_{1}^{2} \leqslant K & +\frac{G M}{\varrho_{1}}-\frac{G M M_{a} M_{b}}{\varrho_{1}^{2}} \frac{r_{*}^{2}}{\varrho_{1}-r_{*}}-\frac{2 G m_{c} g_{2}}{r_{*}} \times \\
& \times\left\{\frac{m_{a}}{M_{b}} \ln \left(1-M_{b} r_{*} / \varrho_{1}\right)+\frac{m_{b}}{M_{a}} \ln \left(1-M_{a} r_{*} / \varrho_{1}\right)\right\},
\end{aligned}
$$

where

$$
\begin{aligned}
K= & \frac{1}{2} \dot{\varrho}_{0}^{2}-\frac{G M}{\varrho_{0}}+\frac{G M M_{a} M_{b}}{\varrho_{0}^{2}} \frac{r_{*}^{2}}{\varrho_{0}-r_{*}}+ \\
& +\frac{2 g_{2} m_{c} G}{r_{*}}\left\{\frac{m_{a}}{M_{b}} \ln \left(\frac{\varrho_{0}+M_{b} r_{*}}{\varrho_{0}}\right)+\frac{m_{b}}{M_{a}} \ln \left(\frac{\varrho_{0}+M_{a} r_{*}}{\varrho_{0}}\right)\right\} .
\end{aligned}
$$

Now, if $\dot{\varrho}>0$ for all $t>t_{0}, \varrho_{1}$ can be made as large as desired by the proper choice of $t$, leading to the expression

$$
\dot{\varrho}_{1}^{2}<0 \quad \text { if } K<0 .
$$

Thus if $K<0$ it must not be the case that $\dot{\varrho}>0$ for all time $t>t_{0}$.

In the expression for $K$, the factor $m_{c} g_{2}$ is $M /\left(m_{a}+m_{b}\right)$, so that the undesirable nature of the expression obtained by Standish (which was

$$
\frac{\dot{\varrho}_{0}^{2}}{2}<\frac{G M}{\varrho_{0}}\left[1-\frac{M_{a} M_{b} r_{*}^{2}}{\varrho_{0}\left(\varrho_{0}-r_{*}\right)}\right]-\frac{Q^{2}}{\varrho_{0}^{2}},
$$

where

$$
Q=\frac{M|\mathbf{L}|}{m_{c}\left(m_{a}+m_{b}\right)}+\left\{\frac{2 G M^{2} M_{a} M_{b} r_{*}}{m_{c}} \times\left[\frac{M_{a} M_{b}}{m_{c}}\left(m_{a}+m_{b}\right)+\frac{r_{*}}{\varrho_{0}}+\frac{M_{a} M_{b} r_{*}}{\varrho_{0}^{2}\left(\varrho_{0}-r_{*}\right)}\right]\right\}^{1 / 2},
$$

and $\mathbf{L}$ is the total angular momentum) with its inverse dependence on $m_{c}$ is not present. However, we have replaced this by the requirement that the two remaining bodies are always sufficiently separated, a requirement that may require numerical integration to check.

Note that

$$
r_{*} \geqslant r \geqslant r_{*} m_{a} m_{b} /\left(m_{a} m_{b}+m_{a} m_{c}+m_{b} m_{c}\right)
$$

gives less variation in the relative positions $m_{a}, m_{b}$ if $m_{c}$ is small than if $m_{c}$ is large. A large mass needs more energy to escape from the system, and hence the variation in the distance between $m_{a}, m_{b}$ can be larger without causing escape.

We are only assured of retention as long as $\dot{\varrho}>0$, and $\dot{\varrho}<0$ together with close passage of $m_{c}$ to one of the other bodies renders the retention theorem invalid.

For motion of the third mass towards the other two, $\dot{\varrho}_{0}$ is negative. We expect $\dot{\varrho}$ 
to increase until, on passage between or close to one or both of the other two masses, $\dot{\varrho}$ changes sign and becomes positive. This change of sign must be accompanied by an instant when $\dot{\varrho}$ is zero. Can we obtain any conditions in the region of close approach? There are difficulties, as the case of collisions presents obvious singularities.

We have from the energy equation

$$
E+F \geqslant 0
$$

or

$$
E \geqslant-G\left(\frac{m_{a} m_{b}}{r}+\frac{m_{a} m_{c}}{\varrho_{a c}}+\frac{m_{b} m_{c}}{\varrho_{b c}}\right) .
$$

If we wish to avoid $\varrho_{a c}, \varrho_{b c}$ becoming infinite (i.e. escape), then we take $E<-G m_{a} m_{b} / r_{*}$ and have

or

$$
G m_{a} m_{b}\left(\frac{1}{r}-\frac{1}{r_{*}}\right)>-G\left(\frac{m_{a} m_{b}}{\varrho_{a c}}+\frac{m_{b} m_{c}}{\varrho_{b c}}\right)
$$

$$
\frac{m_{a} m_{c}}{\varrho_{a c}}+\frac{m_{b} m_{c}}{\varrho_{b c}}>0
$$

For any configuration we know the total energy $E$, and can assert that if $E<$ $<-G m_{a} m_{b} / r_{*}$, then the system is bound, as the mass $m_{c}$ cannot escape. This condition is, of course, less stringent than the retention theorem, but the retention theorem needs to be tested for each $\dot{\varrho}>0, \varrho>r_{*}$ occurrence.

Combining the conditions for escape or retention, we find the region of indeterminancy given by

$$
\begin{aligned}
\frac{-M_{a} M_{b}}{\varrho_{0}-r_{*}}- & \frac{2 \varrho_{0}^{2}}{r_{*}^{3}}\left\{\frac{M_{a}}{M_{b}} \ln \left(1+\frac{M_{b} r_{*}}{\varrho_{0}}\right)+\frac{M_{b}}{M_{a}} \ln \left(1+\frac{M_{a} r_{*}}{\varrho_{0}}\right)\right\} \ll \\
& \leqslant\left(\frac{\dot{\varrho}_{0}^{2}}{2}-\frac{G M}{\varrho_{0}}\right) \frac{\varrho_{0}^{2}}{G M r_{*}^{2}} \leqslant\left(\frac{\varrho_{0}-2 M_{a} M_{b} r_{*}}{\left(\varrho_{0}-M_{a} r_{*}\right)\left(\varrho_{0}-M_{b} r_{*}\right)}\right) M_{a} M_{b} .
\end{aligned}
$$

\section{Containment Theorem}

The procedure used for examination of the possibility of retention may be used to derive a containment theorem. Let us examine the condition that the mass $m_{c}$ does not move further than a distance $R$ from the barycentre of $m_{a}, m_{b}$. We require $\dot{\varrho}_{1}^{2}<0$ for some $\varrho<R$ and can use Equation (2) of Section 2 to give

To ensure return within a sphere of radius $R$ we need

$$
\begin{aligned}
\frac{1}{2}\left(\dot{\varrho}_{1}^{2}-\dot{\varrho}_{0}^{2}\right) \leqslant K+\frac{G M}{R}-\frac{G M M_{a} M_{b}}{R^{2}} \frac{r_{*}^{2}}{R-r_{*}}-\frac{2 G m_{c} g_{2}}{r_{*}}\left\{\frac{m_{a}}{M_{b}} \ln \left(1+M_{b} r_{*} / R\right)+\right. \\
\left.+\frac{m_{b}}{M_{a}} \ln \left(1+M_{a} r_{*} / R\right)\right\} .
\end{aligned}
$$




$$
\begin{aligned}
\frac{1}{2} \dot{\varrho}_{0}^{2}+K+\frac{G M}{R}-\frac{G M_{a} M_{b}}{R^{2}} \frac{r_{*}^{2}}{R-r_{*}} & -\frac{2 G M_{c} g_{2}}{r_{*}}\left\{\frac{m_{a}}{M_{b}} \ln \left(1+M_{b} r_{*} / R\right)+\right. \\
+ & \left.\frac{m_{b}}{M_{a}} \ln \left(1+M_{a} r_{*} / R\right)\right\} \leqslant 0,
\end{aligned}
$$

or

$$
\begin{aligned}
\frac{1}{2} \dot{\varrho}_{0}^{2}+ & \frac{G M}{R \varrho_{0}}\left(\varrho_{0}-R\right)-\frac{G M_{a} M_{b} r_{*}^{2}}{R^{2} \varrho_{0}^{2}\left(R-r_{*}\right)\left(\varrho_{0} r_{*}\right)}\left(\varrho_{0}^{3}-R-r_{*}\left(\varrho_{0}-R\right)\right)- \\
& -\frac{2 G M_{c} g_{2}}{r_{*}}\left\{\frac{m_{a}}{M_{b}} \ln \left(\frac{R+M_{b} r_{*}}{\varrho_{0}+M_{b} r_{*}}\right)+\frac{m_{b}}{M_{a}} \ln \left(\frac{R+M_{a} r_{*}}{\varrho_{0}+M_{a} r_{*}}\right)+\right. \\
& \left.+\left(\frac{m_{a}^{2}+m_{b}^{2}}{m_{a} m_{b}}\right)\left(m_{a}+m_{b}\right) \ln \left(\frac{\varrho_{0}}{R}\right)\right\} \leqslant 0 .
\end{aligned}
$$

\section{CONTAINMENT THEOREM}

If the mutual distance between the bodies of mass $m_{a}$ and $m_{b}$ is bounded by $G\left(m_{a} m_{b}+m_{b} m_{c}+m_{c} m_{a}\right) /|E|=r_{*} \geqslant r \geqslant m_{a} m_{b} r_{*} /\left(m_{a} m_{b}+m_{b} m_{c}+m_{c} m_{a}\right)$ and if at some time $t_{0}$

(i) $\varrho_{0}>r_{*}$,

(ii) $\dot{\varrho}_{0}>0$,

(iii)

$$
\begin{aligned}
\frac{1}{2} \dot{\varrho}_{0}^{2} \leqslant & \frac{G M}{R \varrho_{0}}\left(R-\varrho_{0}\right)-\frac{G M_{a} M_{b} r_{*}^{2}\left(R-\varrho_{0}\right)}{R^{2} \varrho_{0}^{2}\left(R-r_{*}\right)\left(\varrho_{0}-r_{*}\right)}\left(R^{2}+\varrho_{0}^{2}+\varrho_{0} R-r_{*}\right)+ \\
& +\frac{2 G M_{c} g_{2}}{r_{*}}\left\{\frac{m_{a}}{M_{b}} \ln \left(\frac{R+M_{b} r_{*}}{\varrho_{0}+M_{b} r_{*}}\right)+\frac{m_{b}}{M_{a}} \ln \left(\frac{R+M_{a} r_{*}}{\varrho_{0}+M_{a} r_{*}}\right)+\right. \\
& \left.+\frac{\left(m_{a}^{2}+m_{b}^{2}\right)}{m_{a} m_{b}}\left(m_{a}+m_{b}\right) \ln \left(\frac{\varrho_{0}}{R}\right)\right\} .
\end{aligned}
$$

then the mass $m_{c}$ does not move outside a sphere, centred on the barycentre of $m_{a}, m_{b}$ of radius $R$. Again, this theorem only applies to this particular portion of motion, and $m_{c}$ may escape from the sphere after another passage near the centre.

\section{The $n$-Body Problem}

Take $n+1$ bodies, with the possibility of the $(n+1)$ th body being captured by or escaping from the $n$ remaining bodies being of interest.

The Newtonian equations of motion relative to a 'Newtonian origin' $N$ are

$$
\ddot{\mathbf{r}}_{N, i}=-G \sum_{j=1}^{n+1} m_{j} \frac{\left(\mathbf{r}_{N, i}-\mathbf{r}_{N, j}\right)}{\left|\mathbf{r}_{N, i}-\mathbf{r}_{N, j}\right|^{3}}
$$

while the barycentre of the $n$ particles has motion given by 


$$
\left(M-m_{n+1}\right) \ddot{\mathbf{r}}_{N, b}=\sum_{j=1}^{n} m_{j} \ddot{\mathbf{r}}_{N, j},
$$

where $M=\sum_{j=1}^{n+1} m_{j}$

The equation of motion of the $(n+1)$ th particle with respect to the barycentre is

$$
\begin{aligned}
\ddot{\varrho} & =\ddot{\mathbf{r}}_{N, n+1}-\dot{\mathbf{r}}_{N, b} \\
& =-\sum_{j=1}^{n} \frac{m_{j}}{M-m_{n+1}} \ddot{\mathbf{r}}_{N, j} .
\end{aligned}
$$

We know that the centre of gravity $c$ of the $(n+1)$ particles has

$$
\ddot{\mathbf{r}}_{N C}=0 \text {. }
$$

So, as

$$
\begin{aligned}
\mathbf{r}_{N C} & =\sum_{j=1}^{n+1} m_{j} \mathbf{r}_{N, j}, \\
0=\ddot{\mathbf{r}}_{N C} & =\sum_{j=1}^{n+1} m_{j} \ddot{\mathbf{r}}_{N, j},
\end{aligned}
$$

so that

$$
\sum_{j=1}^{n} m_{j} \ddot{\mathbf{r}}_{N, j}=-m_{n+1} \ddot{\mathbf{r}}_{N, n+1},
$$

and

$$
\begin{aligned}
\ddot{\mathbf{Q}} & =\frac{m_{n+1}}{M-m_{n+1}} \ddot{\mathbf{r}}_{N, n+1}+\ddot{\mathbf{r}}_{N, n+1} \\
& =\frac{M}{M-m_{n+1}} \ddot{\mathbf{r}}_{N, n+1},
\end{aligned}
$$

giving

$$
\ddot{\varrho}=-\frac{G M}{M-m_{n+1}} \sum_{j=1}^{n} \frac{m_{j} \mathbf{r}_{j, n+1}}{\left|\mathbf{r}_{j, n+1}\right|^{3}},
$$

where $\mathrm{Q}$ is the radius vector of the $(n+1)$ th body with respect to the barycentre of the remaining $n$ bodies, $\mathbf{r}_{j, n+1}$ the vector between the $j$ th body and the $(n+1)$ th body, $M=\sum_{j=1}^{n+1} m_{j}$. This equation allows for the recoil of the cluster in order to keep the barycentre fixed.

Clearly

$$
\begin{aligned}
\ddot{\varrho} & \geqslant \frac{-G M}{M-m_{n+1}} \sum_{j=1}^{n} \frac{m_{j}}{r_{j, n+1}^{2}} \geqslant \\
& \geqslant \frac{-G M}{M-m_{n+1}} \sum_{j=1}^{n} \frac{m_{j}}{\left(\varrho-r_{*}\right)^{2}}=-\frac{G M}{\left(\varrho-r_{*}\right)^{2}},
\end{aligned}
$$

where $r_{*}$ is the maximum distance of any of the $n$ bodies from the barycentre.

We follow a similar procedure to that in Section 2 for the escape theorem to find $n$-body escape theorem. 
If at some time, $t_{0}$, (i) $\varrho_{0}>r_{*}$, (ii) $\dot{\varrho}_{0}>0$, and (iii) $\frac{1}{2} \dot{\varrho}_{0}^{2}>G M /\left(\varrho_{0}-r_{*}\right)$, then $\varrho \rightarrow \infty$ as $t \rightarrow \infty$ where $M$ is the total mass of the system. Of course, for a spherically symmetric cluster we expect $\frac{1}{2} \dot{\varrho}_{0}^{2}>G M / \varrho_{0}$, so this result is rather weak in a physical sense, but is a rigorous proof for any distribution of matter and velocities.

Using the $\alpha$ notation, $G M /\left(\varrho_{0}-r_{*}\right)$ becomes $1 /(\alpha-1)$, which is clearly a much less stringent condition than that for the three-body problem, unless $\alpha$ is of the order of 10 . This loss is caused by the replacement of $\left(\mathbf{r}_{j, n+1} \cdot \hat{\varrho}\right)$ by $r_{j, n+1}$, so that the angular position of the escaping mass has not been utilized and by the approximation used for $r_{j, n+1}^{2}$ which, in the three-body case, was expanded in terms of Legendre polynomials. A tighter expression for $r_{j, n+1}^{2}$ would strengthen this result, which resembles placing the entire mass of the cluster, the minimum distance away $\left(\varrho-r_{*}\right)$.

However, if we know the form of the distribution of the $n$ particles, we can improve on the estimate of $\ddot{\varrho}$. For example, given $n$ bodies constrained to move along a fixed straight line, then for motion of the $(n+1)$ th body along this line we cannot readily improve on $\varrho \geqslant-G M /\left(\varrho-r_{*}\right)^{2}$ and hence readily improve on the original condition (iii), but for motion of the $(n+1)$ th body perpendicular to the line, if $\theta_{j}$ is given by $\tan \theta_{j}=r_{b, j} / \varrho$ then

$$
\ddot{\varrho}=-\frac{G M}{M-m_{n+1}} \sum_{j=1}^{n} \frac{m_{j} \mathbf{r}_{j, n+1}}{\left|\mathbf{r}_{j, n+1}\right|^{3}},
$$

and

$$
\begin{aligned}
\ddot{\varrho} & \geqslant \frac{G M}{M-m_{n+1}} \sum_{j=1}^{n} \frac{m_{j} \cos \theta_{j}}{\left(r_{j, n+1}\right)^{2}}= \\
& =-\frac{G M}{M-m_{n+1}} \sum_{j=1}^{n} M_{j} \frac{\varrho}{\left(\varrho^{2}+r_{b, j}^{2}\right)^{3 / 2}} \geqslant \\
& \geqslant-G M \frac{\varrho}{\left(\varrho^{2}+r_{*}^{2}\right)^{3 / 2}} .
\end{aligned}
$$

In this case condition (iii) in the escape theorem becomes

$$
\frac{1}{2} \dot{\varrho}_{0}^{2}>G M /\left(\varrho_{0}^{2}+r_{*}^{2}\right)^{1 / 2},
$$

which approximates the spherical case if $r_{*}$ is small, but which for $r_{*}$ large gives less stringent conditions. If the $N$ bodies remain in three groups of mass $M_{1}, M_{2}$ and $M_{1}$ respectively, and can be approximated by three point masses, with $M_{2}$ at the barycentre, then

$$
\ddot{\boldsymbol{Q}}=\frac{-G M}{M-m_{n+1}} \sum_{j=1}^{n} \frac{m_{j} \mathbf{r}_{j, n+1}}{\left|\mathbf{r}_{j, n+1}\right|^{3}},
$$

yields

$$
\ddot{\varrho} \geqslant \frac{-G M}{M-m_{n+1}}\left\{\frac{2 M_{1} \varrho}{\left(\varrho^{2}+r_{*}^{2}\right)^{3 / 2}}+\frac{M_{2}}{\varrho^{2}}\right\},
$$


and condition (iii) is, for motion perpendicular to the line of remaining masses,

$$
\frac{1}{2} \dot{\varrho}_{0}^{2}>\frac{G M}{M-m_{n+1}}\left\{\frac{2 M_{1}}{\left(\varrho_{0}^{2}+r_{*}^{2}\right)^{1 / 2}}+\frac{M_{2}}{\varrho}\right\},
$$

which demonstrates how an increase in separation $r_{*}$ decreases the velocity required for excape.

For the remaining masses constrained to lie in a plane, we would expect escape from the plane to be easier for motion of circularly the $(n+1)$ th body out of the plane. With a symmetric distribution in the plane, motion in the plane gives $\frac{1}{2} \dot{\varrho}_{0}^{2}>G M /\left(\varrho_{0}-r_{*}\right)$, while motion along the line of symmetry perpendicular to the plane gives

$$
\ddot{\varrho} \geqslant \frac{-G M}{M-m_{n+1}} \sum_{j=1}^{n} \frac{m_{j} \varrho}{\left(\varrho^{2}+r_{b, j}^{2}\right)^{3 / 2}},
$$

with $\frac{1}{2} \dot{\varrho}_{0}^{2}>G M /\left(\varrho_{0}^{2}+r_{*}^{2}\right)^{3 / 2}$.

With mass $M_{2}$ at the barycentre and $M_{1}$ distributed in a ring of radius $r_{*}$, motion perpendicular to the plane gives

$$
\ddot{\varrho} \geqslant \frac{-G M}{M-m_{n+1}}\left\{\frac{M_{2}}{\varrho^{2}}+\frac{M_{1} \varrho}{\left(\varrho^{2}+r_{*}^{2}\right)^{3 / 2}}\right\},
$$

with

$$
\frac{1}{2} \dot{\varrho}_{0}^{2} \geqslant \frac{G M}{M-m_{n+1}}\left\{\frac{M_{2}}{\varrho_{0}}+\frac{M}{\left(\varrho_{0}^{2}+r_{*}^{2}\right)^{1 / 2}}\right\} .
$$

For containment we need to extend the energy argument to the $n$-body problem, but unfortunately appear to require additional assumptions.

Clearly

$$
E \geqslant-\frac{1}{2} \sum_{i=1}^{n+1} \sum_{\substack{j=1 \\ j \neq i}}^{n+1} \frac{m_{i} m_{j}}{r_{i j}}
$$

Let the maximum and minimum separation of the $n$ particles be $r_{*}, s_{*}$ respectively

$$
E \geqslant-\frac{1}{2} \sum_{i=1}^{n} \sum_{j=1}^{n} \frac{m_{i} m_{j}}{s_{*}}-m_{n+1} \sum_{i=1}^{n} \frac{m_{i}}{\varrho-r_{*}} .
$$

If $\varrho$ is allowed to become large, $E \geqslant \frac{1}{2} \sum_{i=1}^{n} \sum_{j=1}^{n} m_{i} m_{j} / s_{*}$ and, for $E$ negative and finite

$$
0<s_{*} \leqslant \frac{\sum_{i=1}^{n} \sum_{j=1}^{n} m_{i} m_{j}}{2|E|}
$$

If

$$
s_{*} \geqslant \sum_{i=1}^{n} \sum_{j=1}^{n} \frac{m_{i} m_{j}}{|E|}
$$


$\varrho$ cannot become infinite. This lower bound on the mutual distance between the $n$ bodies ensures that the cluster remains bound. If there is an upper bound $r_{*}$ to the mutual distances, we apparently do not have the restriction, found in the three-body case, that $r_{*}$ necessarily exists for $E$ negative. Escape of more than one body would not be unexpected, however, with these bounds on the distances between the $n$ bodies, we return to the $n$-body form of Equation (1)

$$
\ddot{\varrho}=\mid \boldsymbol{\varrho} \wedge \dot{\boldsymbol{\varrho}}^{\prime 2} \varrho^{3}+g_{2} \partial F / \partial \varrho,
$$

where $g_{2}=M / m_{n+1}\left(M-m_{n+1}\right)$ and $M=\sum_{i=1}^{n} m_{i}$ to find

$$
\begin{aligned}
\ddot{\varrho} & \leqslant \frac{2 g_{2}(E+F)}{\varrho}+g_{2} \frac{\partial F}{\partial \varrho} \leqslant \\
& \leqslant 2 g_{2}\left\{E+\frac{1}{2} G \sum_{i=1}^{n} \sum_{j=1}^{n} \frac{m_{i} m_{j}}{s_{*}}+G \sum_{i=1}^{n} \frac{m_{n+1} m_{i}}{\varrho-r_{*}}\right\} / \varrho+ \\
& +\frac{G M}{M-m_{n+1}}\left[\sum_{j=1}^{n} \frac{m_{j}\left(\mathbf{r}_{j, n+1} \cdot \hat{\varrho}\right)}{r_{j, n+1}^{3}}\right] \leqslant \\
& \leqslant \frac{2 g_{2}}{\varrho}\left\{E+\frac{G}{2} \frac{1}{s_{*}}\left(\sum_{i=1}^{n+1} \sum_{j=1}^{n+1} m_{i} m_{j}\right)+\sum_{i=1}^{n} \frac{m_{n+1} m_{i}}{\varrho-r_{*}}\right\}+ \\
& +\frac{-G M}{M-m_{n+1}}\left[\sum_{j=1}^{n} \frac{m_{j} \mathbf{r}_{j, n+1} \cdot \varrho}{r_{j, n+1}^{3}}\right] \leqslant \\
& \leqslant \frac{2 g_{2} G}{\varrho}\left\{\sum_{i=1}^{n} \frac{\left.\left.\left.m_{n+1} m_{i}\right\}^{\prime}\right\}-\frac{G M}{M-r_{*}}\right\} m_{n+1} \sum_{j=1}^{n} \frac{m_{j}}{r_{j, n+1}^{2}} \leqslant}{} \leqslant \frac{2 g_{2} G}{\varrho} \times \frac{m_{n+1}\left(M-m_{n+1}\right)}{\varrho-r_{*}}+\frac{G M}{M-m_{n+1}} \sum_{j=1}^{n} \frac{m_{j}}{\left(\varrho-r_{*}\right)^{2}}=\right. \\
& =\frac{2 g_{2} G}{\varrho\left(\varrho-r_{*}\right)} m_{n+1}\left(M-m_{n+1}\right)-\frac{G M}{\left(\varrho-r_{*}\right)^{2}} .
\end{aligned}
$$

If $\dot{\varrho}>0$

$$
\begin{aligned}
\dot{\varrho} \varrho & \leqslant \frac{2 g_{2} G m_{n+1}\left(M-m_{n+1}\right)}{\varrho\left(\varrho-r_{*}\right)}-\frac{G M \dot{\varrho}}{\left(\varrho-r_{*}\right)}, \\
\frac{1}{2} \dot{\varrho}^{2}-\frac{1}{2} \dot{\varrho}_{0}^{2} & \leqslant \frac{-2 g_{2} G m_{n+1}\left(M-m_{n+1}\right)}{r_{*}} \ln \frac{\varrho}{\varrho-r_{*}}+\frac{G M}{\left(\varrho-r_{*}\right)}+ \\
& +\frac{2 g_{2} G m_{n+1}\left(M-m_{n+1}\right)}{r_{*}} \ln \frac{\varrho_{0}}{\varrho_{0}-r_{*}}-\frac{G M}{\varrho_{0}-r_{*}}
\end{aligned}
$$

Here $\dot{\varrho}^{2}$ is forced to become zero if

$$
\frac{2 G g_{2} m_{n+1}\left(M-m_{n+1}\right)}{r_{*}} \ln \left(\frac{\varrho_{0}}{\varrho_{0}-r_{*}}\right)-\frac{G M}{\varrho_{0}-r_{*}}+\frac{1}{2} \dot{\varrho}_{0}^{2}<0,
$$


or

$$
\frac{1}{2} \dot{\varrho}_{0}^{2}<\frac{G M}{\varrho_{0}-r_{*}}+\frac{2 G M}{r_{*}} \ln \left(1-r_{*} / \varrho_{0}\right) .
$$

We obtain: $n$-body retention theorem.

If the mutual distances between the $n$ bodies are bounded by

$$
r_{*}>r>s_{*}=\sum_{i=1}^{n} \sum_{j=1}^{n} \frac{m_{i} m_{j}}{|E|},
$$

and if at some time $t_{0}$ (i) $\varrho_{0}>r_{*}$, (ii) $\dot{\varrho}_{0}>0$, (iii)

$$
\frac{1}{2} \dot{\varrho}_{0}^{2}<\frac{G M}{\varrho_{0}-r_{*}}+\frac{2 G M}{r_{*}} \ln \left(1-r_{*} / \varrho_{0}\right),
$$

then the body of mass $m_{n+1}$ does not escape from the system on this particular passage. Subsequent motion after $\varrho<r_{*}$, following $\varrho<0$, may allow escape, and this theorem needs to be re-applied to every $\varrho>r_{*}, \dot{\varrho}>0$ situation. We have not shown that the rest of the cluster does not 'blow up', but include this restriction in the conditions of the theorem.

If the total energy is negative,

$$
\sum_{i=1}^{n+1} \frac{1}{2} m_{i} \mathbf{v}_{i}^{2}-\frac{1}{2} \sum_{\substack{j=1 \\ j \neq i}}^{n+1} \frac{M_{i} M_{j}}{r_{i j}}<0,
$$

and the cluster cannot totally disintegrate. At least one of the distances $r_{i j}$ must be finite.

As for the $n$-body escape theorem, the conditions of the retention theorem can be improved for certain specific mass distributions.

The retention theorem technique may be applied to a capture situation.

Given (i) $\varrho_{0}>r_{*}$, (ii) $\dot{\varrho}_{0}<0$, (iii)

$$
\frac{1}{2} \dot{\varrho}_{0}^{2}<\frac{G M}{\varrho_{0}-r_{*}}+\frac{2 G M}{r_{*}} \ln \left(1-r_{*} / \varrho_{0}\right),
$$

then the body of mass $m_{n+1}$ will either be permanently captured (if $\varrho>r_{*}$ for all time) or will pass closer to the barycentre of the $n$ particles than the distance $r_{*}$.

With $G$ the centre of gravity of the entire system and $B$ the barycentre of the first $n$ particles we have, from the angular momentum integral

$$
\sum_{i=1}^{n+1} m_{i} \mathbf{r}_{N i} \wedge \dot{\mathbf{r}}_{N i}=\text { constant }
$$

Using

$$
\sum_{i=1}^{n+1} m_{i} \mathbf{r}_{G i}=0, \quad m_{n+1}=m
$$




$$
\begin{aligned}
\sum_{i=1}^{n+1} m_{i} \mathbf{r}_{N G} & =\mathbf{a} t+\mathbf{b}, \quad \mathbf{r}_{B, n+1}=\varrho, \\
\sum_{i=1}^{n} m_{i} \mathbf{r}_{B i} & =0, \quad M=\sum_{i=1}^{n} M_{i}, \\
\mathbf{r}_{B i} & =\frac{-\sum_{i=1}^{n} m_{i} \mathbf{r}_{N i}}{m},
\end{aligned}
$$

we find

$$
\begin{aligned}
\sum_{i=1}^{n+1} m_{i} \mathbf{r}_{N i} \wedge \dot{\mathbf{r}}_{N i} & =\sum_{i=1}^{n+1} m_{i} \mathbf{r}_{G i} \wedge \dot{\mathbf{r}}_{G i}= \\
& =\sum_{i=1}^{n+1} m_{i}\left(\mathbf{r}_{G B}+\mathbf{r}_{B i}\right) \wedge\left(\dot{\mathbf{r}}_{G B}+\dot{\mathbf{r}}_{B i}\right) .
\end{aligned}
$$

Now

$$
\begin{aligned}
\mathbf{r}_{G i} & =\mathbf{r}_{G B}+\mathbf{r}_{B i}, \\
0=\sum_{i=1}^{n+1} m_{i} \mathbf{r}_{G i} & =\sum_{i=1}^{n+1} m_{i} \mathbf{r}_{G B}+\sum_{i=1}^{n+1} m_{i} \mathbf{r}_{B i}= \\
& =(M+m) \mathbf{r}_{G B}+\sum_{i=1}^{n} m_{i} \mathbf{r}_{B i}+m \mathbf{Q}= \\
& =(M+m) \mathbf{r}_{G B}+m \mathbf{Q},
\end{aligned}
$$

so the angular momentum is

$$
\begin{aligned}
\sum_{i=1}^{n+1} m_{i} \mathbf{r}_{G B} & \wedge \dot{\mathbf{r}}_{G B}+m \mathbf{Q} \wedge \dot{\mathbf{r}}_{G B}+\mathbf{r}_{G B} \wedge m \dot{\mathbf{Q}}+m \mathbf{Q} \wedge m \dot{\mathbf{Q}}+\sum_{i=1}^{n} m_{i} \mathbf{r}_{B i} \wedge \dot{\mathbf{r}}_{B i}= \\
= & -m \mathbf{Q} \wedge \dot{\mathbf{r}}_{G B}+m \mathbf{Q} \dot{\mathbf{r}}_{G B}-\frac{m \mathbf{Q}}{M+m} \wedge m \dot{Q}+m \mathbf{Q} \wedge \dot{\mathbf{Q}}+\sum_{i=1}^{n} m_{i} \mathbf{r}_{B i} \wedge \dot{\mathbf{r}}_{B i}= \\
& =\frac{m M}{M+m} \mathbf{Q} \wedge \dot{\mathbf{Q}}+\sum_{i=1}^{n} m_{i} \mathbf{r}_{B i} \wedge \dot{\mathbf{r}}_{B i} .
\end{aligned}
$$

In polar coordinates

$$
\begin{aligned}
\varrho^{2} \hat{\theta} & =-\frac{(M+m)}{m M}\left[\mathbf{a} \cdot \hat{\theta}-\sum_{i=1}^{n} \hat{\theta} \cdot m_{i} \mathbf{r}_{B i} \wedge \dot{\mathbf{r}}_{B i}\right] \\
\varrho^{2} \cos \theta \dot{\phi} & =\frac{(M+m)}{m M}\left[a \cdot \hat{\phi}-\sum_{i=1}^{n} \hat{\phi} \cdot m_{i} \mathbf{r}_{B i} \wedge \dot{\mathbf{r}}_{B i}\right] .
\end{aligned}
$$

For $\varrho \rightarrow \infty, \dot{\theta}$ must $\rightarrow 0$ and $\theta \rightarrow \frac{1}{2} \pi$ or $\dot{\phi} \rightarrow 0$.

In other words the escaping particle must eventually be moving away from the system. Note that the origin is moving - as it is the barycentre of the $n$ particles. Reversing time, we must initially 'fire' particles towards system (from infinity!).

The region of indeterminancy of the $n$-body problem is given by 


$$
\frac{G M}{\varrho_{0}-r_{*}}>\frac{G M}{\varrho_{0}-r_{*}}+\frac{2 G M}{r_{*}} \ln \left(1-r_{*} / \varrho_{0}\right) .
$$

The use of velocity of escape of the form $\frac{1}{2} v_{\infty}^{2}=G M / r_{*}$ is criticized by Kurth (1957) who states that

It is ... usually assumed that a star with this or with a higher energy will actually leave the system.... It is doubtful whether this procedure is reliable. The motion of the star in the course of time also depends, for example, on its initial direction and, to a large extent, on the motions of the remaining stars.

In this paper I derive rigorous conditions for escape, which also show that the usual assumption of a velocity of escape is valid, provided that the remaining cluster is bounded in space for all time.

It is also known (Jacob's criterion of stability) that a gravitating system is unstable if its total energy $E$ is positive. Kurth (p. 65) states

up to the present no satisfactory criterion has been found, for systems with negative total energy, to decide between the two possibilities of periodicity and disintegration. It appears to be one of the most important unsolved problems in the mechanics of stellar system to discover such a criterion....

Kurth also states that (p. 61) if the mass centre of the system is taken as origin, then $\sum_{i=1}^{n} M_{i} r_{i}=0$ shows that if one particle escapes to infinity, at least another and most probably two will escape to infinity. Hence if a system disintegrates, at least three bodies will, as a rule, escape to infinity. This conclusion is not valid if, as one particle escapes from the cluster, the rest of the cluster en masse 'escapes to infinity', but remains bound together. Such a motion will satisfy the preceding equation, but will from the point of view of an external observer, present the picture of a single particle escaping from a moving system.

\section{Acknowledgements}

I thank Drs Myles Standish and Aarseth for their comments, together with those of two anonymous referees. This research was aided by grant NRC A6529 (Canada).

\section{References}

Chazy, J.: 1922, Ann. Sci. École Norm. Sup. 39.

Griffith, J. S. and North, R. D.: 1974, Celes. Mech. 8, 473.

Kurth, R.: 1957, Introduction to the Mechanics of Stellar Systems, Pergamon, p. 92.

Standish, E. M.: 1971, Celes. Mech. 4, 44.

Standish, E. M.: 1972, Celes. Mech. 6, 352.

Tevzadze, G. A.: 1962, Izv. Akad. Nauk Armyan SSR 15, No. 5, p. 67.

Yoshida, J.: 1972, Publ. Astron. Soc. Japan 24, 391.

\section{DISCUSSION}

$S$. J. Aarseth: It is of interest to note that this type of escape criterion is sharper than is usually required for numerical studies. Thus the computations of three-body systems by Szebehely showed in every case that all escaping particles satisfied both the simple two-body criterion discussed here, when using $r_{*}=15$. 